\title{
Complementarity of Input Devices to Achieve Knowledge Sharing in Meetings
}

\author{
Himanshu Verma, Flaviu Roman, Silvia Magrelli, Patrick Jermann, Pierre Dillenbourg \\ CRAFT, École Polytechnique Fédérale de Lausanne (EPFL) \\ Switzerland \\ \{himanshu.verma, flaviu.roman, silvia.magrelli, patrick.jermann, pierre.dillenbourg\}@epfl.ch
}

\begin{abstract}
In co-located meetings, participants create and share content to establish a common understanding. In this paper, we present a collaborative environment that enables group members to create and share content simultaneously by providing them with different kinds of individual input devices and a shared workspace. We also report on an exploratory study to investigate the influence of the input device used on the shared knowledge produced by the group. The results suggest that driven by the affordances, various input devices complement each other. We thus recommend groups to be equipped with multitude of them to support diverse meeting task demands. Additionally, we observed that groupware usage differs across various phases of the problem-solving activity. This provides implications for the design of collaborative environments to assist each of the respective phases of the task, in order to extend their usefulness for the group.
\end{abstract}

\section{Author Keywords}

Single display groupware; co-located collaboration; shared workspaces; multiple input devices; $\mathrm{CSCW}$

\section{ACM Classification Keywords}

H.5.3 Information Interfaces and Presentation: Group and Organizational Interfaces-Computer-supported cooperative work.

\section{General Terms \\ Human Factors; Experimentation.}

\section{INTRODUCTION}

Meetings are a widespread way of decision making and problem solving in a collaborative way. In order to support the construction of shared understanding, participants create artifacts such as written notes, drawings, annotations and so forth. However, content creation is often a distributed activity as attendees have their own copy of notes, and they are not aware of their peers' actions. In order to support an idea or to

Permission to make digital or hard copies of all or part of this work for personal or classroom use is granted without fee provided that copies are not made or distributed for profit or commercial advantage and that copies bear this notice and the full citation on the first page. To copy otherwise, or republish, to post on servers or to redistribute to lists, requires prior specific permission and/or a fee.

CSCW'13, February 23-27, 2013, San Antonio, Texas, USA.

Copyright 2012 ACM 978-1-4503-1209-7/13/02...\$15.00. express their opinion, participants share these artifacts among each other. Sharing requires either the transfer of artifacts to others or explicit acquisition of control over the shared resources such as a flip-chart or a whiteboard. Transfer of artifacts is sometimes inconvenient and the shared information is only visible to a subset of group members who are in proximity to the artifact. This may lead to reduced visibility of the artifact within the group. In addition, acquisition of shared resources by a participant could lead to floor monopolization. Also, whiteboards are often used in meetings to facilitate sharing of content, but the shared content over white-boards is not permanent, cannot be replicated and is hard to modify [26].

As our research is oriented towards co-located brainstorming and meetings, we hypothesize that a Single Display Groupware (SDG) as defined by Stewart et al. [27] can mitigate these issues relating to sharing and creation of content in meetings. Kaplan et al. [14] identified that technologies allowing for collective manipulation and group awareness (which are supplied by a SDG) support co-located collaboration. A SDG embodies the benefits of a shared workspace coupled with simultaneous interaction ability that is enabled by multiple input devices - one for each user. Presence of a shared workspace assists group members to collaborate over a common task at the same time. In addition, supplying each group member with an input device of her own makes sharing more probable and helps in avoiding situations where a user claims ownership over the shared resources, thus moderating floor monopolization. Further, the affordances of the input device also influence the kind of activity best supported by the device. For example, a keyboard is a convenient way of inputting text to a computer, while a pen is more effective for drawing schemas and figures on paper or white-board. Therefore, it is crucial to leverage these factors into the design of a collaborative meeting environment which can facilitate the auxiliary activity of content creation and sharing.

In this paper, we present a collaborative environment to support content creation and sharing in meetings for small groups. The system incorporates a shared wall-mounted workspace where users can interact using either mouse and keyboard or pen and paper. We also present an exploratory user study comparing the two input configurations, to understand the role of input devices on the way users share content within the group and how this can influence the task outcome. 


\section{RELATED WORK}

Many researchers have studied SDGs in diverse scenarios ranging from education of school children [18] to organizational meetings [12, 13]. Dillenbourg et al. [4] identified that shared workspaces as comprised in SDG, offer potential benefits such as increased awareness, facilitate the process of grounding, and could act as group memory due to the persistence of information shared over it. Further, large wall-mounted displays such as whiteboards facilitate the fluid transitions between individual and group work [28], as well as enable shared visualization and give an overview of the shared content [9]. Considering the simultaneous interaction ability (as enabled by SDG), Rogers et al. [19] observed more balanced participation within a group in terms of verbal utterances, when each group member was supplied with her own input entry point. Besides, SDG was observed to positively affect the collaboration among children due to the increased awareness about the shared artifacts and fellow group members. In a study conducted by Scott et al. [21], an increase in children's participation, engagement level and enjoyment of the activity was observed, while using a SDG to solve a collaborative puzzle task.

However, existing SDG systems are restricted in the way users can interact with the system. For example, the interaction with the shared display is limited to individual claimed spaces in Dynamo [12] and users have to compete for the screen space in order to share. In PointRight [13] the interaction itself is restricted due to technological constraints and the system allows only one mouse pointer per display, thus reducing chances of collaboration. Also, some SDG systems require multi-touch tables to interact with the system as in WeSpace [33]. This can be cumbersome to use, as shared content on the table has reduced visibility among the group mainly due to orientation of the users around the table [23] and occlusion [22]. Moreover, the space over the multi-touch tables is meant for interaction and cannot be used to lay physical objects [10]. Furthermore, their prolonged usage results into fatigue [32].

In addition, these systems require users to learn the functionality of the system and perform extra effort every time to keep it operable, such as connecting individual laptops or PDAs to share content $[12,26,33]$. This additional effort required in proportion to a small collective benefit, motivates users to revert back to conventional means of collaboration using paper and flip-charts, and thus causes the groupware to fail in its purpose as emphasized by Grudin [5].

Despite the presence of varied systems supporting meetings, pen and paper are still preferred as they are a quicker way of expressing an idea or making a point. Perennial research has been done to leverage the pen based interaction into interfaces supporting collaboration. For example, NoteLook [2], NotePals [3] and We-Met [34] use stylus over PDA and tablets to enable note taking during meetings, while in NiCE Discussion Room [6] group members can use pen and paper to create and share content. NotePals [3] is a system to share meeting notes among the group using a shared note repository, however the interaction is restricted due to the small size

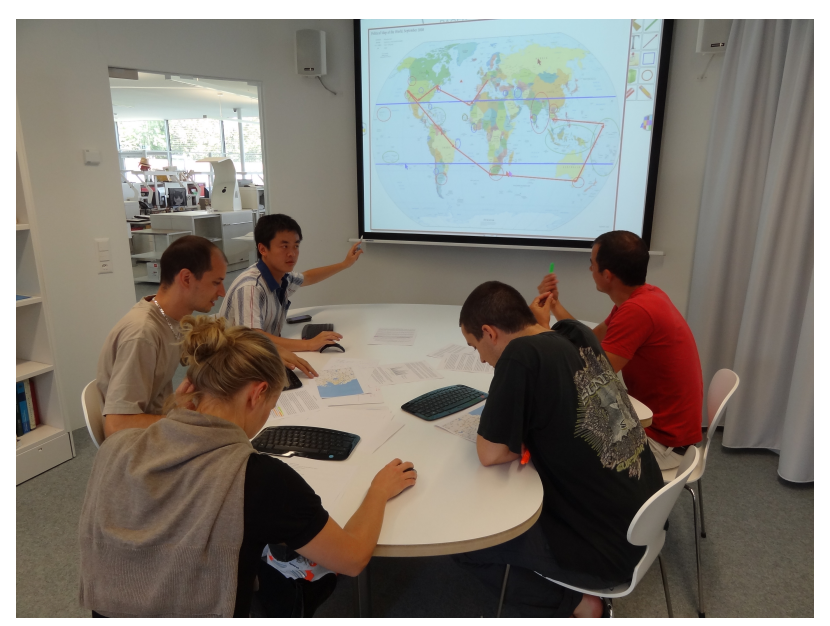

Figure 1: Collaborative environment with the meeting table in the Mouse and Keyboard setup.

of the PDA screen. NoteLook [2] facilitates users to generate meeting minutes by annotating directly over the meeting images (extracted from the video cameras in meeting room and signifying meeting activity and important events), but is not suitable for brainstorming where group members frequently generate ideas in the form of drawings or schemas. The pen and paper interface in NiCE Discussion Room [6] does not allow multiple users to work simultaneously on a single problem. Wolf et al. [34] observed that pen based interfaces are unobtrusive and do not interfere with interpersonal communications during collaboration. This suggests that an obtrusive input device increases the extraneous cognitive load of the user resulting in attentional shift from the collaborative task to the device usage.

Researchers have also studied the impact of various aspects of groupware on the collaborative processes in face-to-face meetings. Inkpen et al. [11] conducted an ergonomic field study to investigate the influence of seating arrangement, orientation of group members, size and number of displays on co-located collaboration. In addition, Mandryk et al. [16] studied the effects of privacy and user's proximity to the display on the collaborative processes. The results suggest that varied display types have different affordances: horizontal displays are perceived to be more natural and comfortable for co-located collaboration, whereas vertical displays offer a better perspective on the shared information. Seating arrangement around the table was found to influence the distribution of roles within the group in face-to-face setting [24]. Likewise, orientation around the table was observed to affect the coordination, communication and comprehension among the group members [15]. Furthermore, Ryall et al. [20] have examined the effects of table size and group size on group interactions around a single, shared-display groupware. The results suggest that group size affects the way digital shared content was organized and used by the group members. 


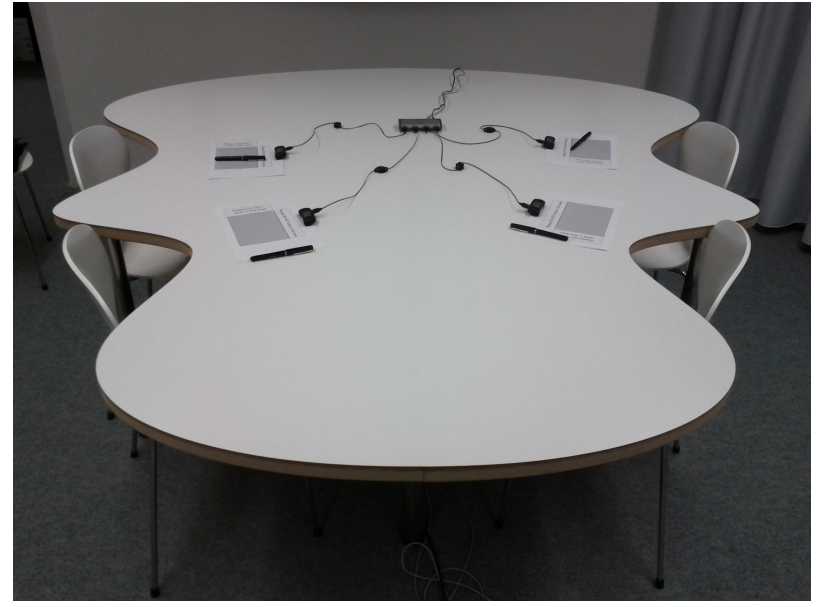

Figure 2: Meeting table in the Pen and Paper setup.

\section{TECHNICAL SETUP}

Our proposed system design incorporates a front projected display of $2 \mathrm{~m} \times 2 \mathrm{~m}$ size, as well as a meeting table placed in front of the public display (see Figure 1). The meeting table has a specific shape (wave-like shape as shown in Figure 2) designed to ergonomically facilitate face-to-face interaction between participants as well as interaction with the public display. The system consists of two setups - in the first setup each user is equipped with a wireless mouse and keyboard (MK, Figure 1), while in the other each user is supplied with a digital pen ${ }^{1}$ and paper (PP, Figure 2). Each input device is color coded, which helps users identify their respective cursors on the public display in both the setups. The public display as well as the input devices are connected to a single computer.

\section{Design Rationale}

Wallace et al. [32] have established that single-display groupware (SDG) is beneficial for coordinating over the shared resources whereas multi-display groupware (MDG) is advantageous for individual task duties. This was our initial design decision to select SDG over MDG. Tang et al. [29] have demonstrated that collaborative coupling (both loose and tight coupling) between participants during meetings is influenced by the physical arrangement of the meeting place. This led us to the wave-like design of the meeting table (see Figure 2), which can ergonomically facilitate smooth transitions between different coupling styles as well as transitions between individual and group activities.

Next, we chose mouse and keyboard as the preferable input devices instead of multi-touch tables. Mice offer a higher degree of pointing accuracy and keyboards are a faster, convenient and robust means of entering text as compared to touchbased or on-screen keyboards [8]. Furthermore, a comparison between multi-mouse SDG and multi-touch tables suggests that users are more efficient with the multi-mouse SDG, but

\footnotetext{
${ }^{1}$ IRISNotes Pen : www.irisnotes.de
}

prefer the multi-touch setup [7]. During the iterative development of our system, we tested it in several meetings and brainstormings in our lab. We experienced that mouse was a very efficient device to move, edit and delete objects (text and images). However, the mouse was not very efficient when users quickly wanted to express an idea by drawing a schema or a diagram, as the users had to change between different tools frequently (for example from lines to circle). Even though we chose to provide users with the minimal number of tools required to create and share content, the mouse was not found to be easily compatible with the real time dynamics of a meeting. Furthermore, using a mouse for the purpose of drawing increased the extraneous cognitive load of the users and thus made it hard for them to focus on the ongoing collaboration simultaneously while using the mouse.

This led us to use pen and paper in our collaborative system as creation of content such as drawings and schemas is considerably quicker and more efficient using a pen instead of a mouse [34]. Further, availability of multiple pens during meetings (one for each user), will reduce the chances of a user acquiring explicit control over the shared resources, thus moderating floor monopolization. Terrenghi et al. [30] point out that tangibility of an artifact and the surrounding space allows for easy manipulation of the artifact. As pen and paper are tangible as compared to digital objects over multi-touch table, we decided to use the former for our collaborative meeting environment.

\section{Shared Workspace over Public Display}

Similar to the conventional paper-based flip charts, we use a page metaphor for our shared workspace. Our shared workspace allows multiple users to simultaneously add and manipulate artifacts such as graphics, text and images. In case of space shortage on the current page, users can create a new blank page while concurrently saving the previous work. In the mouse and keyboard setup, the group is provided with a Page Manager widget designed to enable the group to browse through previously created pages (see the top part of Figure 3a).

In the pen setup, users are provided with normal A4 sheets upon which is marked the area mapped to the entire public display. Anything written within this area is concurrently reflected on the public display in the color of the device color code (see Figure 3b). Changing of page on the public display is accomplished by tapping the pen over one of the two squares printed on the top left corner of the paper indicating the direction of the page change.

\section{Tool Collection}

We wanted to provide users only with the minimal functionality necessary to support the task at hand, as abundance of tools consume a lot of screen space and involves higher anticipated learning efforts and time. This might result in an increase in extraneous cognitive load and would leave less cognitive capacity for the execution of the task; i.e. bringing the content creation and sharing (which is a supporting activity going on in the background) to the forefront and push the 


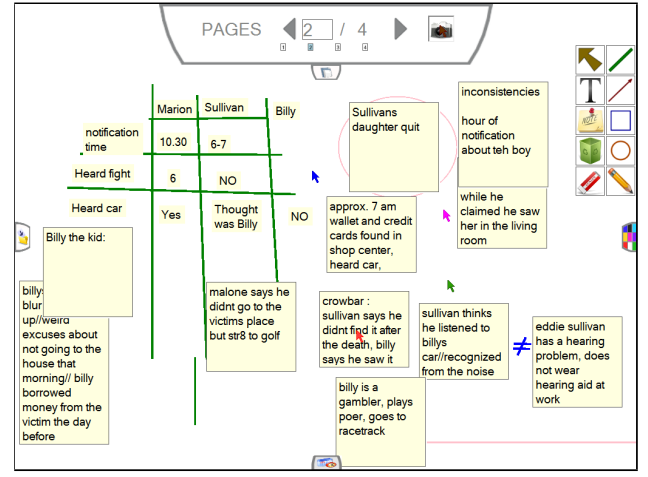

(a) Mouse and Keyboard Condition

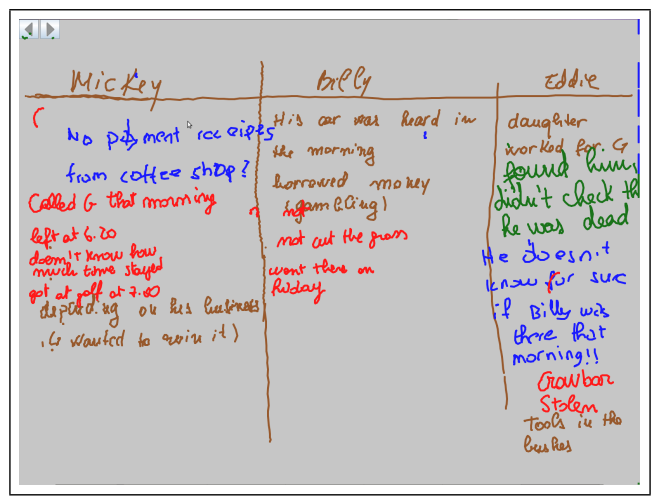

(b) Pen and Paper Contition

Figure 3: Snapshot of the shared workspace in MK condition (top) and PP condition (bottom).

collaborative task in the background. In the mouse and keyboard setup the basic toolset includes lines, arrows, ellipse, rectangle, free-hand drawing and post-its. The users are provided with two identical toolsets containing the above mentioned functionalities; one of the toolbars is displayed next to each mouse pointer, the other one is public for everyone to use (see the top right corner of Figure 3a). In this setup, users can arrange the digital content (post-its and images) over the shared workspace by simply dragging the object from one place to another, as well as stack artifacts on top of others. Furthermore, any user can annotate and manipulate the digital artifacts created by other group members; thus enabling group members to simultaneously work on the same problem without requiring to divide it into sub-problems.

In the pen system no additional tools are provided to the user, like an ability to create predefined shapes from strokes on the paper or moving artifacts over the public display. The pen is intended to be used in the natural way.

\section{USER STUDY}

We hypothesized that affordances of an input device may render it appropriate for a specific type of interaction. Thus, users might perceive one input device as an efficient (and faster) way of creating one type of shared representation than another. For example, a keyboard might more often be used to create textual elements and pen for drawing elements, while a mouse is more efficient to move and organize artifacts. In order to investigate the differences between the input devices, and how this difference influences the kind of shared representations created by groups, we conducted an exploratory user study.

\section{Participants}

Sixty-six participants (23 females and 43 males) aged 1735 years (average age of 23) were recruited for the study, from the Swiss Federal Institute of Technology, Lausanne. The participants were all university students studying various engineering domains. Groups of 4 participants passed the experiment task, except for two cases where the experiment was performed with a group of 3 participants (both groups in the pen and paper condition). The participants were randomly assigned to the groups across the two conditions. There were 11 mixed-gender groups, 2 groups were all female groups and 4 were all male groups. Regarding the familiarity among the subjects, there were 7 (out of 17) groups where at least 2 participants were acquainted with each other. However, to increase familiarity between all participants, we asked each group member to introduce themselves before each experiment. In addition, 2 subjects (out of 66) reported themselves to be colorblind, one for red and green pair and the other for blue colors. Participants were paid 25 Swiss Francs each for their participation in the study.

\section{Experimental Task}

During the experiment, each group was asked to complete the murder mystery task designed by Stasser et al. [25], where group members were required to use the collaborative environment to generate and share content. It is a hidden profile task where some crucial information about the suspects is unshared among the group members. The correct identification of the murderer requires the group members to combine the information by sharing and discussing as much as possible, as incomplete information may lead to inferior solutions and premature solution convergence [17]. Furthermore, as identified by Mennecke [17], group members share more information in situations where they are explicitly made aware about the unshared information. We informed each group about the hidden pieces of information (but not the location) in the experiment booklets (containing various interviews with the characters of the story) at the start of the experiment.

This task was chosen because it resembles a collaborative problem solving strategy common to meetings where the group has some shared knowledge about the task as well as some unshared information which could prove to be important towards the end result. In addition, the choice of the input device to create and share information using a groupware might influence the sharing behavior of participants. Groups using different collaborative tools (mouse and keyboard (MK) versus pen and paper (PP)) might have different shared representations of the murder mystery. For example, group members in the MK condition might share facts and link them, while groups in the PP condition might come up with the timeline of the murder. 


\section{Procedure}

Firstly, the group members were welcomed and asked to choose their preferred seat around the table. Once seated, the group members were asked to complete a pre-experiment questionnaire recording basic demographic data and were requested not to change their seating position, as this information was also recorded in the questionnaire. Next, the group was introduced to the collaborative system which they were about to use (either mouse and keyboard or pen and paper), and they were given some time to try out the system and familiarize themselves with the tools. After this, the experiment booklets were provided to the group members, and the group was asked to complete the task by determining the most likely suspect to have committed the crime. The group members were then asked to complete a post-experiment questionnaire recording their perception about the groupware usage and collaboration. Finally, after disclosing the true identity of the murderer and the motives involved in the study, all the subjects were given the compensation and were thanked for their participation.

\section{Design}

We used a two input configurations (mouse and keyboard $(\mathrm{MK})$ versus pen and paper (PP)), between-subjects design for our user study. Groups of 4 participants completed the experimental task in one of the two conditions. The task was not time bound, but an upper limit of 2 hours was set and the groups were asked to suggest the name of the suspect most likely to have committed the crime. However, the average time required to complete the task by all the groups was 90 minutes. In total, there were 17 groups during the study (15 groups with 4 participants, 2 groups with 3 participants). Nine groups completed the experiment in MK condition and eight groups in PP condition.

\section{Data Collection}

Each participant was asked to complete two questionnaires; one before and one after the study. The pre-experiment questionnaire collected basic information about participants including working preferences (if the subject prefers to work alone or in groups), weekly time spent in meetings and type of meetings attended. We also asked if the subject suffered from colorblindness and of what kind, because the content created over the shared workspace by different group members is represented in different colors corresponding to the input device color code.

The post-experiment questionnaire recorded participant's perception of the task, the emerging social structure, the usage of the collaborative tool and the purpose for which the tool was used. This questionnaire contained statements that the participant had to rate on a 5-point Likert scale, with 1 as strong disagreement and 5 as strong agreement. The information related to the task concerned the perception of a participant about the group's consensus on the solution, time sufficiency and difference in opinion among the group members. The statements about the emerging social structure relate to the presence of a group leader, individual's perception about participation and acceptance of her contributions by the group. Further, the usage information in the questionnaire records effort required to create artifacts (text, drawings and linking information) and effort required to coordinate. Finally, the questionnaire recorded the perceived purpose of the collaborative tool: whether the group used the tool to collect facts, make hypotheses about the suspects or recreate the story line. Space was also provided for the users to add some open ended comments.

In addition, two observers manually recorded the number of deictic gestures and utterances for each participant in the group. The utterances were classified either to be relating to the task or to the organization of group activities. Similarly, deictic gestures were divided so as to refer to the task material (booklets given to the participants) or to the content shared on the public display. Further, all the interactions with the collaborative environment were recorded in system log files.

\section{Research Hypotheses}

We hypothesize that affordances of an input device render it appropriate for a specific kind of task activity. As a pen is more efficient and quick to draw figures or schemas and a keyboard is a faster way of entering text, we expect that there will be more drawing elements in the PP condition (H1a), and more textual elements in the MK condition $(\mathbf{H 1 b})$. Furthermore, as more users simultaneously share content in the PP setup, and due to the absence of visual feedback from other users on the paper, there will be more chances of content overlapping (or coordination breakdowns). However, in the MK setup as users directly look at the shared workspace while interacting, there will be less overlap of content (due to location coordination) and coordination may be easier (H2).

In addition, the difference in the input mechanism can also lead to different shared representations of the problem solving task being produced by the group members. For example, as entering text is faster using a keyboard and users are accustomed to moving objects (files, folders etc.) on their desktop using a mouse, the MK setup can be used to collect facts about the suspects. On the contrary, as drawing is quicker with a pen on paper, we assume that the group members will produce a more conceptual representation of the murder mystery such as a timeline of the murder.

\section{RESULTS AND ANALYSES}

\section{Input Devices}

An analysis of variance with task time as the dependent and experiment condition as the independent variable reveals that groups in the PP condition completed the task in significantly less time as compared to the MK condition $(\mathrm{F}(1,15)=11.70$, $\mathrm{p}<.01)$. One possible explanation for this could be that group members are more efficient with pen and paper. But this observation does not inform us about the amount of content created. Next, we look at the type of content created by the group members; i.e. the amount of textual elements and drawing elements generated. The number of textual elements corresponds to the number of words generated by each participant in each group during the experiment. Similarly, a count was made for the drawing elements, where each drawing stroke (line, ellipse, etc.) was counted as a single drawing element. A significant difference was found in the amount of 
text generated using the groupware across the two conditions, with more text generated in the MK condition $(\mathrm{F}(1,64)=4.08$, $\mathrm{p}=.04)$. Thus, our hypothesis $\mathrm{H} 1 \mathrm{~b}$ is statistically validated. On the contrary, more drawing elements were created in the PP condition, but this difference was not found to be statistically significant and therefore there is not enough statistical evidence to validate hypothesis H1a. Also, no significant difference was found in the number of pages created across the two conditions.

Regarding the questionnaire data about the subject's perception of the effort required to create artifacts, we observed that it required significantly more effort to create text in the PP condition $(\mathrm{F}(1,63)=4.30, \mathrm{p}=.04)$. On the other hand, creating drawing elements was perceived as significantly easier in the $P P$ condition $(F(1,62)=5.71, p=.02)$. This goes in line with the aforementioned observation that larger number of textual elements were created in the MK condition. In addition, this effort might also suggest that users perceive keyboard as an efficient way of inputting text, and pen as a quicker way of creating drawing elements. Thus, indicating that the affordances of an input device in usage might influence a specific task activity.

Next, considering the effort required to avoid overlapping of content over the shared workspace (i.e. avoiding coordination breakdowns), the participants responded that it was easier to coordinate in the PP setup. However, this difference is not statistically significant. This observation is contrary to our hypothesis $\mathrm{H} 2$, and a possible explanation for this could be the lack of transparency of the post-its and text objects in the MK setup. Also, these text objects automatically adjust their size depending on the quantity of text contained, as more text is added the post-its grow in size possibly occluding the artifact under it as indicated by Zanella and Greenberg in [35].

\section{Task Outcome}

Considering the success (or failure) of a group across the two conditions (i.e. identifying the correct suspect as the murderer), we observed that there was no significant difference $\left(\chi^{2}=0.04, \mathrm{p}>.05\right.$, Kruskal-Wallis Test). However, success in hidden profile tasks is influenced by other factors as well, such as the information the group is aware of, effective sharing of information, motivation of the group members and the importance of information perceived by them [25]. Different input techniques can only assist to facilitate the process of effective sharing. In addition, as indicated by Mennecke [17], enabling groups to share information simultaneously reduces the chances of production blocking (i.e. lack of opportunity for group members to share ideas they have created). But, this parallelism in sharing might also impede groups from discovering the true hidden profile as the individuals fail to consume the information shared by the group (see Figure 4b), i.e. consumption blocking. In other words, as shown in Figure $4 \mathrm{~b}$ if group members create and share information using the groupware for the most part of the meeting without pausing to discuss and assimilate this information, it might result in a bad decision quality or failure in the task. This suggests that groups should follow phases of sharing and phases of assimilation (with very few actions on the shared workspace) of

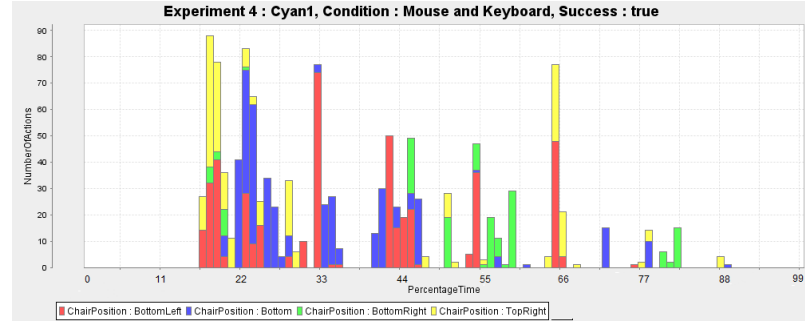

(a) Success in Task

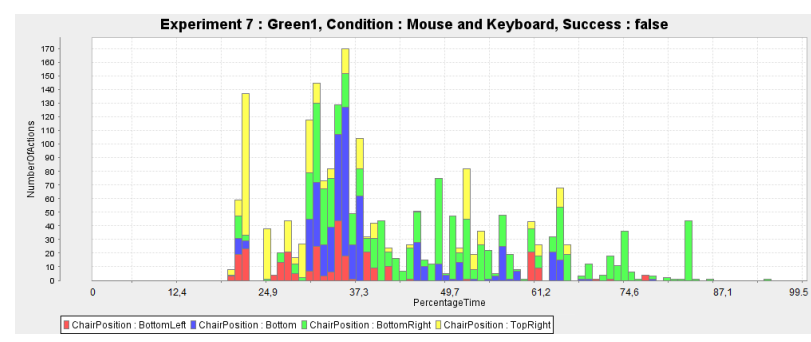

(b) Failure in Task

Figure 4: Activity map for each subject (in different color) in a group during the experiment. The $\mathrm{X}$-axis represents the elapsed time in percentage, and the Y-axis represents the number of actions.

the information in order to achieve success (Figure 4a). However, we did not observe such a pattern while looking at the activity maps of all the successful groups.

Furthermore, we observed that successful groups produced more utterances but this difference was not statistically significant. On the contrary, significantly less gestures were made towards the public display $\left(\chi^{2}=12.86, \mathrm{p}<.001\right.$, KruskalWallis Test) as well as towards the experiment material $(\mathrm{F}(1,64)=4.32, \mathrm{p}=.04)$ by the successful groups. Also, significantly higher number of pages were created by the successful groups $\left(\chi^{2}=8.51, \mathrm{p}<.01\right.$, Kruskal-Wallis Test $)$, which confirms that effective sharing is essential for success in hidden profile tasks. Taking into consideration the Likert scale value denoting the difference in opinions among group members regarding the murderer, we observed that members of successful groups were significantly more differing in their opinions $(\mathrm{F}(1,64)=27.29, \mathrm{p}<.001)$. This corroborates the fact that difference in opinion results into dialogue among the group members, and thus making the true discovery of hidden profile highly probable. This dialogue might also provide the appropriate platform to reconsider the problem from a completely different viewpoint.

\section{Shared Knowledge Representations}

The experiment booklets distributed among the group members contained interviews between detectives, suspects and other characters. Few excerpts from these interviews were left unshared in a way that only one group member had this information, while the others didn't. In order to correctly identify the murderer, the group members should unify the initially shared (common to all the group members) as well as the initially unshared (specific to each user) information. Ac- 


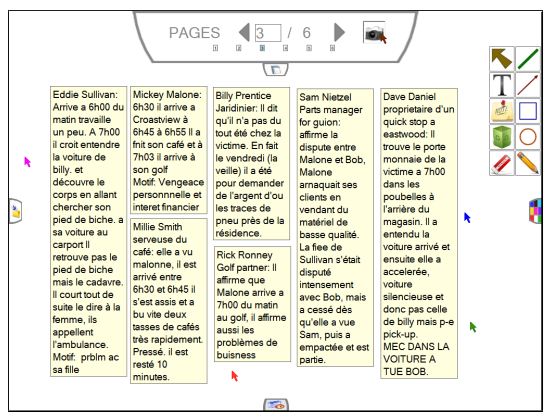

(a) MK: Facts

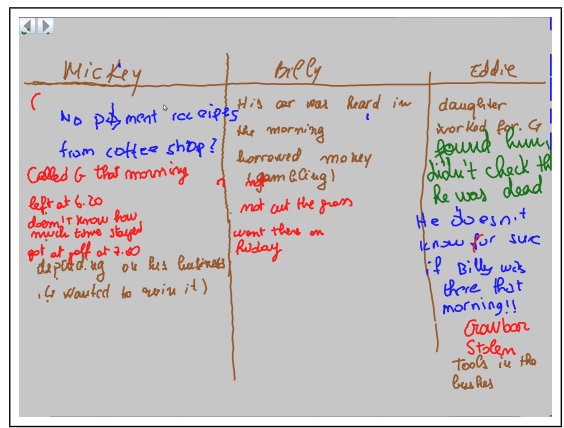

(d) PP: Facts

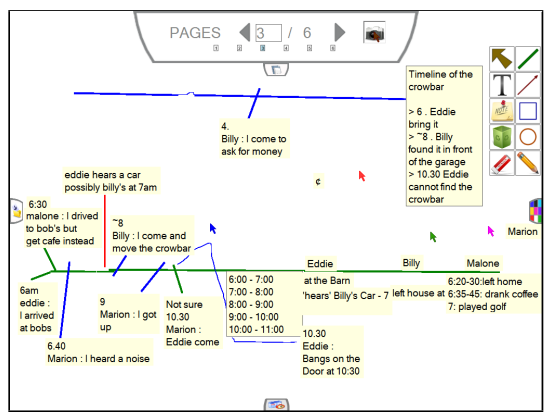

(b) MK: Timeline

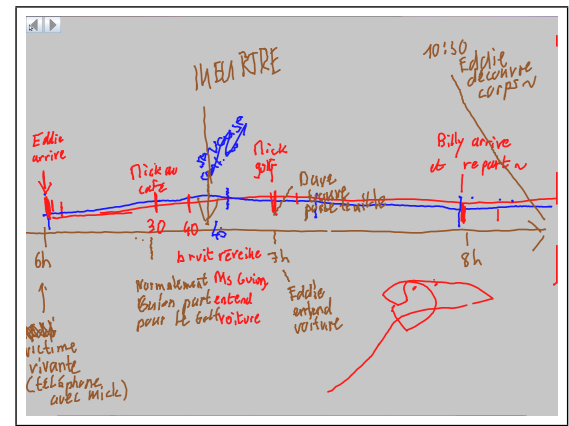

(e) PP: Timeline

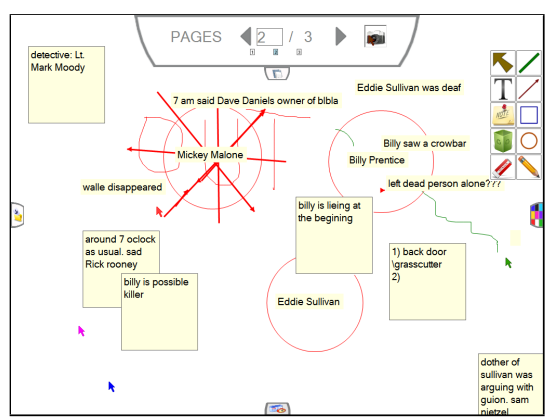

(c) MK: Syntheses

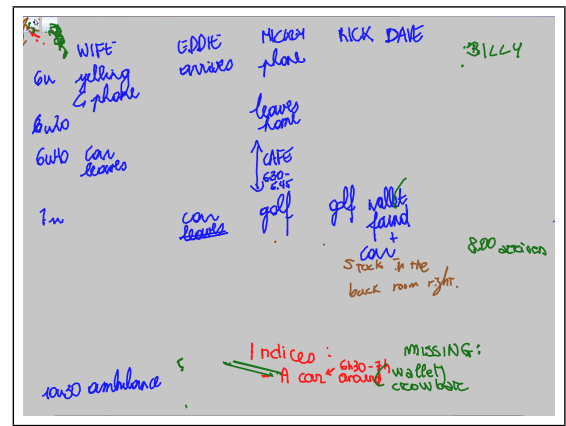

(f) PP: Syntheses

Figure 5: Classification of the shared content over the public display.

complishing this requires that group members simultaneously collect facts about the suspects, connect and associate the shared facts to create the narrative. During the experiments, we observed that groups created similar kinds of knowledge representations over the shared display in both the conditions. We can briefly classify the shared knowledge representations into three categories - facts (Figures 5a \& 5d), timelines (Figures $5 \mathrm{~b} \& 5 \mathrm{e}$ ) and syntheses (Figures $5 \mathrm{c} \& 5 \mathrm{f}$ ).

Facts were collected mostly during the starting phase of the experiment while reading the experiment booklets. The group members tended to partition the available space over the shared workspace; in each partition facts about one of the suspects or characters was collected as shown in Figures 5a and $5 \mathrm{~d}$. In the MK setup, different post-its denoted the separation of one character from another (Figure 5a), and with time the facts were added to the appropriate post-it by the group members. On the other hand, in PP setup one group member took the initiative to partition the space manually after discussing on the number of partitions (Figure 5d). In some cases, the group members skipped (the fact collection phase) directly to a more schematic representation such as a timeline or a grid. In order to do so, the group members showed each other their experiment booklets to find the differences in the text, thus reducing their effort to share a lot of information and sharing only the crucial episodes of the story.

The timelines were usually made towards the end of the reading phase, and mark the phase where group members start assimilating the facts. The timelines in both the conditions, looked more or less the same as shown in Figures $5 \mathrm{~b}$ and $5 \mathrm{e}$.
In the MK condition, a timeline was made by situating the post-its at appropriate positions (Figure $5 \mathrm{~b}$ ) followed by linking these post-its to the horizontal timeline. Whereas in the PP condition, group members wrote directly at the exact spot (see Figure 5e). However, we observed that only one group (out of 9 groups) created a timeline in the MK condition. On the contrary, many such representations were made in the PP condition. A possible explanation for this could be that drawing in the MK condition consumed a lot of time, as users frequently have to change modes while writing text, drawing lines and even to drag post-its from one position to another. However, drawing was much faster in the PP condition, as the users simply have to write on the paper while looking at the public display to coordinate.

Syntheses representations were usually created during the convergent phase of the discussion, where users were considering the key episodes of the story and debating about the most likely suspect. In this representation the shared workspace served as a temporary storage of relevant facts which could assist the group in reaching a decision. In the MK condition, the syntheses resembled a screen with randomly distributed textual facts which were later assigned to different clusters or concepts as the discussion progressed (Figure 5c). The clusters were represented by explicitly drawing circles to contain related facts, or by placing post-its over one another with slight overlap. Similarly, in the PP condition, participants browsed for the relevant facts from the previous pages and created a hybrid representation of crucial episodes and links (see Figure 5f). 
Considering the typology of produced content by the groups, we see a transition between individual sharing activity and collaborative information processing as observed by Tang et al. in [28] in case of whiteboards. The group members start with collecting information as facts and figures, and towards the end of the discussion build a more global knowledge about the story. This important group dynamics is probably driven by the transitions between these shared representations created by the group.

\section{Task Efficiency and Input Devices}

Analysis of experiment data reveals that groups created more text in the MK condition and more drawings in PP condition. In addition, mouse in MK condition allows moving, deleting and editing objects which is not possible in PP condition. These observations can possibly be attributed to the participant's efficiency with an input device. Also, the affordances of an input device favor the usage of the device for one kind of task activity than another. Pen might prove to be a faster way of expressing ideas as a schema as compared to a mouse. Similarly, keyboard could be a faster (and convenient) way of inputting large quantity of text as compared to pen.

In order to confirm that an input device is good for a specific task activity, we conducted a micro study with 10 colleagues from our lab. Each subject was asked to create text and drawing elements in both the conditions. For the text creation activity two short stories of exactly the same length ( 100 words each) were chosen, and the participants were asked to use the keyboard (in MK condition) and pen (in PP condition) to write these stories. The time taken by the participant was recorded while using the keyboard and the pen. Similarly for the drawing activity, each participant was asked to play tictac-toe with oneself under a constraint that the match should end in a draw. This constraint ensures that the participants do not randomly fill in the cells but use cognition to carefully place the move so that no one wins. This is to ensure that the task is of the same difficulty for the subject across the two conditions in within-subject design. The time taken by the participant was again recorded while performing the task using a pen (PP condition) and a mouse (MK condition).

We used a two input configurations, within-subject design for this micro study. The conditions as well as the activities were counterbalanced. A Paired t-test was performed over the task completion time of subjects in the text and drawing activity, across the two conditions. The results of the analysis reveal that subjects were significantly quicker and more efficient in creating text using a keyboard as compared to pen $(\mathrm{t}(9)=2.87$, $\mathrm{p}=.01$ ). On the other hand, subjects took significantly less time to create drawings using a pen as compared to mouse $(\mathrm{t}(9)=5.15, \mathrm{p}<.001)$. The analysis indicates that keyboard is an effective way of creating text as compared to pen, whereas pen is much quicker in a drawing task than a mouse. In addition, mouse is suitable for activities requiring higher pointing accuracy, such as dragging, selecting objects and changing modes. Also, the observations from this micro study confirm with the previous observations regarding different type of content produced across the two conditions.

\footnotetext{
${ }^{2}$ IRISNotes Digital Pen
}

This study suggests that the affordances of the input device in usage can influence the kind of content created and shared while using a single display groupware. Further, as group members choose to create a specific type of shared content, they would preferably go for the input device which is efficient, fast and highly convenient for such a representation, and this decision seems to be made implicitly by the user and does not involve cognitive load. This might explain why only one group (out of 9) created the timeline in MK condition, whereas more timelines were created in the PP condition. For such a representation, mouse was a very slow alternative for drawing lines and consumed a lot of time, therefore the groups preferred not to make timelines. However, in the PP condition as participants can draw lines and link facts quickly, this representation was more frequent in this condition.

\section{Differences in Group Composition}

Considering the familiarity among subjects and its influence on the group processes across the two conditions (MK and $\mathrm{PP}$ ), we performed a Chi-Square test to study if previouslyacquainted subjects were distributed differently across the conditions. The results demonstrate that there is no significant difference in distribution $\left(\chi^{2}(1, N=66)=1.25, \mathrm{p}=.26\right)$. Next, results of Chi-Square test demonstrates that there are significantly fewer than expected females assigned to the MK condition than to the PP condition $\left(\chi^{2}(1, N=66)=4.40\right.$, $\mathrm{p}=.03)$. This might appear as a bias in our user study, consequently we investigated if the amount of shared content produced (number of textual elements and drawing elements) and participation (utterances and gestures) varied with gender. Results of a mixed-effect ANOVA show that no significant difference was found in the amount of text generated $(\mathrm{F}(1,64)=0.92, \mathrm{p}=.34)$ and the amount of drawing elements generated $(\mathrm{F}(1,64)=0.04, \mathrm{p}=.84)$ across gender. This signifies that the amount of shared content produced is not influenced by gender or familiarity among subjects. Furthermore, we found no significant gender differences in the number of gestures towards the shared workspace $(\mathrm{F}(1,63)=0.08, \mathrm{p}=.78)$ as well as towards the experiment booklets $(F(1,64)=0.46$, $\mathrm{p}=.5$ ). Likewise, the number of utterances made by the group members were not statistically different across gender $(\mathrm{F}(1,64)=0.28, \mathrm{p}=.59)$. Consequently, we can conclude that gender did not play a significant role in group processes.

\section{Groupware Usage Transition and Leadership}

Our observations of the various shared representations produced by the group on the shared workspace at different points in the task (as previously discussed), show a transition from primarily factual content towards a conceptual shared representation. An examination of the concurrent usage (number of participants simultaneously interacting with the system) of the collaborative environment, exhibits similar transition. We observed that all the groups used the collaborative environment concurrently (more than one participants interacting simultaneously) during the divergent or information collection phase of the group work. However, the collaborative environment was used in a single-user mode during the discussion or convergent phase of task activity, where one group member took the responsibility of interacting with the 
system while others chose to be part of the ongoing discussion. Ryall et al. [20] observed a similar transition from an individual (or parallel) activity mode to a collective mode of operation during task for groups with 4 participants. Our observation either implies that roles emerged within the group, or indicates the problems related to the ergonomics of the collaborative environment.

Regarding the emergence of roles during the task, we consider only one role of a group leader. After each experiment the two observers were asked to rate (on a 5-point Likert scale) each group member on having the qualities of a group leader. The participant in each group with the highest average rating from both the observers was identified as a group leader. However there might be a limitation in this method of specifying a group leader, as the observers might have different perception about leadership. Moreover, the results of regression analysis demonstrate that group leaders interacted $12.67 \%$ more with the shared workspace (number of actions including creating text and drawing as well as moving artifacts) than other group members $(\mathrm{t}(48)=2.63, \mathrm{p}=.01)$. In addition, group leaders had significantly higher number of utterances (19.28 utterances) than other subjects $(\mathrm{t}(47)=3.15$, $\mathrm{p}<.01)$. Further, higher number of gestures towards shared workspace ( 3 gestures, $\mathrm{t}(47)=2.73, \mathrm{p}<.01$ ) as well as gestures towards experiment booklets ( 7.49 gestures, $t(48)=4.06$, $\mathrm{p}<.001$ ) were observed for group leaders. These results indicate that group leaders were speaking and gesturing more, as well as took the responsibility of interacting with the system during the convergent phase of task (which is also demonstrated by the analysis of system logs).

Further, in order to investigate if problems with the ergonomics of the system resulted in one subject taking control of the system, we analyzed if the seating position around the table (or orientation with the shared workspace) influenced the emergence of leadership. The result of the Chi-Square test exhibits that there is no significant difference in leadership across the different seating positions around the table $\left(\chi^{2}(4, N=66)=5.14, \mathrm{p}=.27\right)$.

These observations emphasize on a very important group dynamics of task validation and coordination. The convergent phase of the task (information assimilation and decision making) requires relatively higher coordination among the group members as well as multiple perspectives to the shared knowledge. As a result, one of the group members acquires the control of the groupware and interacts with it while simultaneously validating (with other group members) each interaction with the shared content (such as annotating or organizing crucial facts about the suspects). These phases in group activity are consistent with the ones indicated by McGrath et al. [1] and Tschan [31]. In addition, we see a transition from a multi-user mode to a single-user mode as shown in Figure 6, where each group transits into the single-user mode by sliding down what we call a Cliff of Convergence. The cliff of convergence denotes the transition from the divergent phase (center of Figure 6) to the convergent phase (right side of Figure 6), whereas the region on the left side of Figure 6 denotes the phase where group members familiarize themselves with

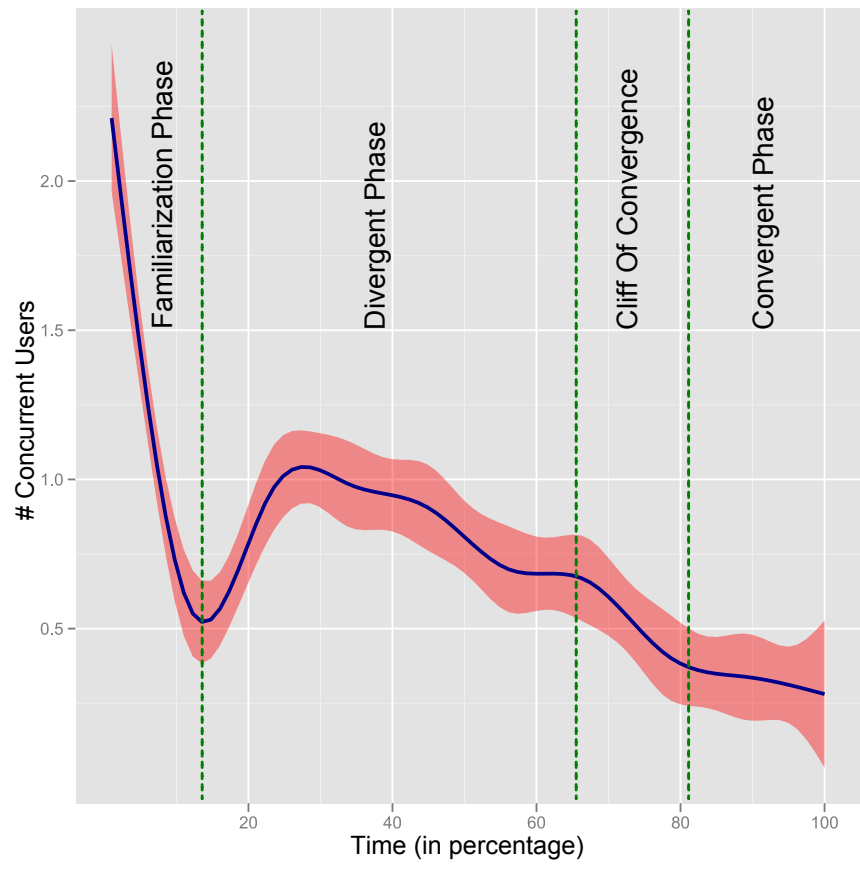

Figure 6: Moving average plot with confidence interval. Xaxis represents the normalized time for all the groups and the Y-axis represents the number of users concurrently using the system.

the system before the task starts. Most existing SDGs assist only in the divergent phase of group activity by facilitating content creation and sharing. However, the existence of this phenomenon suggests that groups would benefit from SDGs that also support the convergent phase of activity.

\section{Factor Analysis}

In order to check if few experiment variables correlate well with each other, so that they can be aggregated and meaningful analysis can be made with them, we performed a factor analysis. We separated the experiment variables into two sets before performing the factor analysis. The first set regards the collaboration (or teamwork) among the group members, while the second set considers the groupware usage (or task work) during the task. This division was made based on the classification suggested by Wallace et al. [32]. The experiment variables in the first set concern the perceived participation of the individual in the group activity, opinion about the task outcome and the observed participation of the subject (utterances, gestures etc.). The second set of variables include participant's perception about effort required to create content and coordinate, content shared by the user and the perceived purpose of the system.

The first factor analysis shows the emergence of two main dimensions, namely active participation and group acceptance. Experiment variables concerning the utterances, gestures and the groupware usage are significantly correlated with the active participation dimension, whereas participants 
preferring to work alone are strongly anti-correlated. Furthermore, variables recording the participant's perception about the task outcome and individual's role in the group are correlated to the group acceptance dimension. We observe that group members who preferred working in groups, participated actively during the task and had higher number of utterances, gestures and shared more information. On the contrary, subjects preferring individual work had less participation in the group. Further, the individuals whose contributions were accepted by the group tended to participate actively and vice versa. This observation emphasizes on a very important group dynamic of trust building within the group. As the group acknowledges the contributions of an individual, the individual recognizes a higher level of involvement in the group and thus continues making contributions. Also a higher level of engagement of the group members could be related to the feeling that the group converged to a common consensus. Finally, the difference in opinions of the group members about the murderer might lead to higher acceptance of contributions by the group. Also as mentioned previously, the members of successful groups were differing in their opinions about the murderer. These results could provide a convincing evidence for the characteristic of successful groups. During the task the group members had different opinions about the outcome, resulting in dialogues and negotiations which finally led to a better quality decision.

The second factor analysis provides some interesting aspects about groupware usage. Experiment variables relating to the coordination aspects of the groupware usage are found to be strongly correlated to one dimension, referring to the effort required by individuals to coordinate the task activity. We observe that, if it is hard to coordinate using a groupware (more coordination breakdowns due to the overlapping of shared information on the public display or group members simultaneously using the same part of the display), the groupware is not used for a conceptual representation of the murder mystery, such as a timeline of important events. Rather the groupware is used more as a group memory to collect and store factual information about the suspects. From our previous observation we see that as it is easier to coordinate in the PP condition as compared to MK condition, more timelines were created in the PP condition than in the MK condition. In addition, significantly more amount of text was created in the MK condition. These results point out that the PP setup was used by the groups to create conceptual representation of the murder story, whereas the MK setup was used more often to collect facts. This is again coherent with the analysis of different shared representations from the previous section. The perceived affordances of an input device influence the user's choice of one input device over another for the collaborative task, which in turn affects the type of shared representations produced.

\section{DISCUSSION}

The collaborative meeting environment presented in this article was designed to assist the content creation and sharing activity in co-located meetings of small groups. Prior research has suggested that SDGs positively influence the collaboration $[21,32]$. We conducted a user study to investigate the effects of different input techniques on the way participants share knowledge about the task. Our experiment findings provide some important implications towards the design of single display groupware. In problem solving meetings, creation and sharing of content is not a main activity but a supporting activity for the task at hand. During the task, group members can choose to create diverse representations of their shared knowledge depending on the task demands. For example, a group discussing about a murder mystery (as in our user study) might choose to make a timeline, whereas a group designing a database might go for a relational schema. Therefore, considering that different tasks demand varied representations, it is impractical to restrict the interaction with the groupware by choosing only one input mechanism. In other words, if group members are supplied with just one type of input device to interact, it limits the number of shared representations which could possibly be produced by the group.

The experiment results clearly indicate that there exists a mapping between the input device affordances and the knowledge representation which the task demands. Presence of multiple kinds of input devices for each user provide the user with an opportunity to select the input mechanism most appropriate to adequately support the task at hand; i.e. an ecology of input tools capable of supporting various kinds of content representations. In other words, instead of supplying users with just one input mechanism (such as mouse and keyboard $O R$ pen $O R$ just multi-touch table), the group members might wish to be equipped with more than one kind of interaction techniques. So, when there is a demand for a specific shared knowledge representation, the group members make the mapping between the perceived affordance of the suitable input device and the task demand. We call this phenomenon opportunistic sharing, as the mapping between the input device and shared representation is made opportunistically by the user herself.

An analysis of the artifacts shared by the groups lead us to classify them into three kinds based on the time when they were created, visual differences and the immediate purpose the content served. We observed that during the whole task, groups created many representations. The classification exhibits some important characteristic of the shared workspace (wall mounted public display); the role of the shared workspace and the transitions in the collaborative coupling of the group members. During the task the shared workspace is used by the group members to offload their cognitive load (group memory) by sharing information. At the later stage this information is processed, conceptualized and comprehended by the group and acts more as the group knowledge which leads to decision making. Regarding the collaborative coupling, we see a transition from loosely coupled group (individual task of sharing and surfacing facts) to a more tightly coupled group where the group members build a shared understanding. These observations are in line with the research done by Tang et al. in [28, 29]. Additionally, we observed that such a transition happened only once during the task, whereas one can expect such transitions to occur several times during the problem solving activity. However, these transitions might depend on the type of activity and cer- 
tain activities might have transitions during each iteration (or phase) of the group activity.

Further analyses of the groupware usage shows that the group members used the collaborative environment concurrently during the information collection phase, whereas during the decision making phase only a single group member used the groupware. This transition from a divergent phase of the group activity to the convergent phase happens over a Cliff of Convergence, after which the interactions with the shared workspace are done only by the group leader while others prefer to be part of the ongoing discussion. This observation implies that SDGs will better assist groups (or group leaders) during the convergent phase of the task if they provide tools or agents which facilitate the organization of shared content in formats that enable discussion about it. This is an important design implication as existing SDGs are situated in the divergent phase of group activity requiring creation and sharing of content.

Concerning the coordination of the task activity while using the groupware, the factor analysis provides interesting results. The effort required by the users to coordinate defines the purpose of the collaborative environment. If it requires a lot of effort to coordinate, the groupware is used as a group memory to store information which could be referred later during the task. In such a situation, the collaborative environment is regarded disconnected with the task by the group and becomes invisible to the group as the task progresses. On the other hand, the easier it is to coordinate, the more tightly coupled is the group activity with the collaborative environment and the shared workspace is at the center of discussion. Further, the factor analysis emphasizes some crucial aspects of collaboration and group dynamics, and is explained by the emergence of two factors namely active participation and group acceptance.

In our user study we did not extensively test for the differences in the types of users and their behaviors across the two input configurations. In addition, participant's preferences for input devices were not accounted for in the analyses, as a keyboard (and mouse) and a digital pen do not have the same prevalence in our sample population. This is because most of the subjects were not familiar to the digital pen, and consequently preference would not be informed by experience but rather by novelty.

\section{CONCLUSION}

We have presented a collaborative meeting environment to support content creation and sharing in co-located meetings, which enables group members to concurrently interact with the shared workspace while mitigating issues such as floor monopolization. The collaborative environment is composed of a shared wall-mounted workspace and two different input configurations - mouse and keyboard AND pen and paper along with a meeting table by which the setup is supported. We have also conducted an exploratory user study to investigate the effect of difference in input configurations on the way group members create and share content. We observe that different input devices complement each other with regard to the shared representations that can be produced using these input devices, where one kind of content can be conveniently produced with one input configuration. We conclude that, group members should be equipped with varied input devices so that they can opportunistically map the adequate input device to the representation demanded by the task. We call this phenomenon opportunistic sharing. Finally, we observed that the usage of the groupware varies with the phase of the problem solving activity and this provides important implications for the design of collaborative environments to assist each of the respective phases of the task.

\section{ACKNOWLEDGMENTS}

First of all, we would like to thank the anonymous reviewers for their comments and suggestions, which greatly helped us to improve the paper. Next, we would like to thank Cristián Alcoholado Moenne and Olivier Guédat for their contribution to the development of this system. Thanks to all the students who volunteered to observe the meeting sessions, also special thanks to all the students who participated in the study. Finally, we would like to thank the Swiss Post for supporting our research.

\section{REFERENCES}

1. Arrow, H., McGrath, J. E., and Berdahl, J. L. Small Groups as Complex Systems: Formation, Coordination, Development, and Adaptation. Sage Publications, Inc., 2000.

2. Chiu, P., Kapuskar, A., Reitmeier, S., and Wilcox, L. NoteLook: Taking notes in meetings with digital video and ink. In Proceedings of the seventh ACM international conference on Multimedia (Part 1), MULTIMEDIA '99, ACM (1999), 149-158.

3. Davis, R. C., Landay, J. A., Chen, V., Huang, J., Lee, R. B., Li, F. C., Lin, J., Morrey, III, C. B., Schleimer, B., Price, M. N., and Schilit, B. N. NotePals: Lightweight note sharing by the group, for the group. In Proceedings of the SIGCHI conference on Human factors in computing systems: the CHI is the limit, CHI '99, ACM (1999), 338-345.

4. Dillenbourg, P., and Traum, D. Does a shared screen make a shared solution? In Proceedings of the 1999 conference on Computer support for collaborative learning, CSCL '99, International Society of the Learning Sciences (1999).

5. Grudin, J. Why CSCW applications fail: Problems in the design and evaluation of organizational interfaces. In Proceedings of the 1988 ACM conference on Computer-supported cooperative work, CSCW'88, ACM (1988), 85-93.

6. Haller, M., Leitner, J., Seifried, T., Wallace, J. R., Scott, S. D., Richter, C., Brandl, P., Gokcezade, A., and Hunter, S. The NiCE Discussion Room: Integrating paper and digital media to support co-located group meetings. In Proceedings of the 28th international conference on Human factors in computing systems, CHI '10, ACM (2010), 609-618. 
7. Hansen, T. E., and Hourcade, J. P. Comparing multi-touch tabletops and multi-mouse single-display groupware setups. In Proceedings of the 3rd Mexican Workshop on Human Computer Interaction, MexIHC '10, Universidad Politécnica de San Luis Potosí (2010), 36-43.

8. Hartmann, B., Morris, M. R., Benko, H., and Wilson, A. D. Augmenting interactive tables with mice \& keyboards. In Proceedings of the 22nd annual ACM symposium on User interface software and technology, UIST'09, ACM (2009), 149-152.

9. Hilliges, O., Terrenghi, L., Boring, S., Kim, D., Richter, H., and Butz, A. Designing for collaborative creative problem solving. In Proceedings of the 6th ACM SIGCHI conference on Creativity \&amp; cognition, C\&amp;C '07, ACM (New York, NY, USA, 2007), 137-146.

10. Hinrichs, U., Hancock, M., Collins, C., and Carpendale, S. Examination of text-entry methods for tabletop displays. In Horizontal Interactive Human-Computer Systems, 2007. TABLETOP '07. Second Annual IEEE International Workshop on (Oct. 2007), $105-112$.

11. Inkpen, K., Hawkey, K., Kellar, M., Mandryk, R. L., Parker, R., Reilly, D., Scott, S., and Whalen, T. Exploring display factors that influence co-located collaboration: Angle, Size, Number and User Arrangement. In Proceedings of HCI International, Lawrence Erlbaum Associates (2005), 22-27.

12. Izadi, S., Brignull, H., Rodden, T., Rogers, Y., and Underwood, M. Dynamo: A public interactive surface supporting the cooperative sharing and exchange of media. In Proceedings of the 16th annual ACM symposium on User interface software and technology, UIST '03, ACM (2003), 159-168.

13. Johanson, B., Hutchins, G., Winograd, T., and Stone, M. PointRight: Experience with flexible input redirection in interactive workspaces. In Proceedings of the 15th annual ACM symposium on User interface software and technology, UIST '02, ACM (2002), 227-234.

14. Kaplan, F., DoLenh, S., Bachour, K., Kao, G. Y.-i., Gault, C., and Dillenbourg, P. Interpersonal computers for higher education. In Interactive Artifacts and Furniture Supporting Collaborative Work and Learning, P. Dillenbourg, J. Huang, and M. Cherubini, Eds., vol. 10 of Computer-Supported Collaborative Learning Series. Springer US, 2009, 1-17.

15. Kruger, R., Carpendale, S., Scott, S. D., and Greenberg, $S$. How people use orientation on tables: comprehension, coordination and communication. In Proceedings of the 2003 international ACM SIGGROUP conference on Supporting group work, GROUP '03, ACM (New York, NY, USA, 2003), 369-378.

16. Mandryk, R. L., Scott, S. D., and Inkpen, K. Display factors influncing co-located collaboration. In In Conference Supplement to ACM CSCW'02 Computer Supported Cooperative Work (2002), 137-138.
17. Mennecke, B. E. Using Group Support Systems to discover hidden profiles: An examination of the influence of group size and meeting structures on information sharing and decision quality. International Journal of Human-Computer Studies 47, 3 (1997), 387 405 .

18. Moraveji, N., Inkpen, K., Cutrell, E., and Balakrishnan, R. A Mischief of Mice: Examining children's performance in Single Display Groupware systems with 1 to 32 mice. In Proceedings of the 27th international conference on Human factors in computing systems, CHI '09, ACM (2009), 2157-2166.

19. Rogers, Y., Lim, Y.-k., Hazlewood, W. R., and Marshall, P. Equal Opportunities: Do shareable interfaces promote more group participation than single user displays? Human-Computer Interaction 24, 1-2 (2009), 79-116.

20. Ryall, K., Forlines, C., Shen, C., and Morris, M. R. Exploring the effects of group size and table size on interactions with tabletop shared-display groupware. In Proceedings of the 2004 ACM conference on Computer supported cooperative work, CSCW '04, ACM (New York, NY, USA, 2004), 284-293.

21. Scott, S., Mandryk, R., and Inkpen, K. Understanding children's collaborative interactions in shared environments. Journal of Computer Assisted Learning 19, 2 (2003), 220-228.

22. Shen, C., Ryall, K., Forlines, C., Esenther, A., Vernier, F. D., Everitt, K., Wu, M., Wigdor, D., Morris, M. R., HancocK, M., and Tse, E. Collaborative tabletop research and evaluation. In Interactive Artifacts and Furniture Supporting Collaborative Work and Learning, P. Dillenbourg, J. Huang, and M. Cherubini, Eds., vol. 10 of Computer-Supported Collaborative Learning Series. Springer US, 2009, 1-17.

23. Shen, C., Vernier, F. D., Forlines, C., and Ringel, M. DiamondSpin: An extensible toolkit for around-the-table interaction. In Proceedings of the SIGCHI conference on Human factors in computing systems, CHI '04, ACM (2004), 167-174.

24. Sommer, R. Small group ecology. Psychological Bulletin 67, 2 (Feb 1967), 145-152.

25. Stasser, G., and Stewart, D. Discovery of hidden profiles by Decision-Making groups: Solving a problem versus making a judgment. Journal of Personality and Social Psychology 63, 3 (1992), 426-434.

26. Stefik, M., Foster, G., Bobrow, D. G., Kahn, K., Lanning, S., and Suchman, L. Beyond the Chalkboard: Computer support for collaboration and problem solving in meetngs. Commun. ACM 30 (January 1987), 32-47.

27. Stewart, J., Bederson, B. B., and Druin, A. Single Display Groupware: A model for co-present collaboration. In Proceedings of the SIGCHI conference on Human factors in computing systems: the CHI is the limit, CHI '99, ACM (1999), 286-293. 
28. Tang, A., Lanir, J., Greenberg, S., and Fels, S.

Supporting transitions in work: Informing large display application design by understanding whiteboard use. In Proceedings of the ACM 2009 international conference on Supporting group work, GROUP '09, ACM (2009), 149-158.

29. Tang, A., Tory, M., Po, B., Neumann, P., and Carpendale, S. Collaborative coupling over tabletop displays. In Proceedings of the SIGCHI conference on Human Factors in computing systems, CHI '06, ACM (2006), 1181-1190.

30. Terrenghi, L., Kirk, D., Sellen, A., and Izadi, S. Affordances for manipulation of physical versus digital media on interactive surfaces. In Proceedings of the SIGCHI conference on Human factors in computing systems, CHI '07, ACM (2007), 1157-1166.

31. Tschan, F. Ideal cycles of communication (or cognition) in Triads, Dyads, and Individuals. Small Group Research 33, 6 (Dec 2002), 615-643.
32. Wallace, J., Scott, S., Stutz, T., Enns, T., and Inkpen, K. Investigating teamwork and taskwork in single- and multi-display groupware systems. Personal and Ubiquitous Computing 13 (2009), 569-581.

33. Wigdor, D., Jiang, H., Forlines, C., Borkin, M., and Shen, C. WeSpace: the design development and deployment of a walk-up and share multi-surface visual collaboration system. In Proceedings of the 27th international conference on Human factors in computing systems, CHI '09, ACM (2009), 1237-1246.

34. Wolf, C. G., Rhyne, J. R., and Briggs, L. K. Communication and information retrieval with a pen-based meeting support tool. In Proceedings of the 1992 ACM conference on Computer-supported cooperative work, CSCW '92, ACM (1992), 322-329.

35. Zanella, A., and Greenberg, S. Reducing interference in single display groupware through transparency. In $E C S C W$ 2001, W. Prinz, M. Jarke, Y. Rogers, K. Schmidt, and V. Wulf, Eds. Springer Netherlands, 2002, 339-358. 